\title{
Equivalencia y valor en procesos de reciprocidad e intercambio entre los mapuches
}

\author{
Equivalence and value in processes of reciprocity \\ and exchange between Mapuche people
}

\author{
Julio Tereucán-Angulo / julio.tereucan@ufrontera.cl \\ Claudio Briceño-Olivera / claudio.briceno@ufrontera.cl \\ José Gálvez-Nieto / jose.galvez@ufrontera.cl \\ Universidad de La Frontera, Chile
}

\begin{abstract}
Reciprocity and exchange are two issues of interest in anthropology stated in Marcel Mauss' essay about gifting. However, this topic has had limited attention among the Mapuche, especially within the area of social practices that people and domestic groups perform in rural communities. The objective of this article is the analysis of reciprocity and exchange from the perspective of interchangeability and associated processes in Mapuche communities, to later evaluate the principles involved, especially the ones related to the equivalence and value of reciprocity; postulating that based on the analysis the following can understood: practices of social organization, economic relations, everyday social interactions between domestic groups, among other areas. The research was conducted with a qualitative strategy, and the ethnographic method was developed to process data collection in Mapuche communities of southern Chile.
\end{abstract}

Key words: reciprocity, interchange, equivalence, value, Mapuche people.

Resumen: La reciprocidad y el intercambio constituyen un tema de tratamiento en la antropología a partir del ensayo sobre los dones de Marcel Mauss; no obstante, esta temática ha tenido un escaso tratamiento entre los mapuches, especialmente desde la óptica de las prácticas sociales que personas y grupos domésticos realizan en las comunidades rurales. El objetivo del artículo es analizar la reciprocidad y el intercambio desde la perspectiva de la intercambiabilidad y procesos asociados en comunidades mapuches, para posteriormente dimensionar los principios involucrados, en particular los referidos a equivalencia y valor en la reciprocidad; postulando que a partir del análisis de estas prácticas se puede entender y comprender la organización social, la dinámica de relaciones económicas, las interrelaciones sociales cotidianas entre grupos domésticos, entre otros ámbitos. La investigación es de tipo cualitativa bajo el método etnográfico y se realizó en comunidades mapuches del sur de Chile.

Palabras clave: reciprocidad, intercambio, equivalencia, valor, mapuches. 


\section{Introducción}

La dinámica y proceso del don y/o el intercambio constituye un aspecto importante en la vida social, cultural y económica de muchos grupos; y en disciplinas como la antropología social, sociología y economía ha tenido diversas y extensas discusiones a partir del trabajo de Marcel Mauss (1971), quien describe un ciclo de prestaciones formado por tres obligaciones: dar - recibir - devolver, cuyo objetivo principal es el establecimiento y mantenimiento de relaciones de solidaridad entre los grupos que componen una sociedad (Bordieu, 2007; Kowalski, 2011).

Diversos autores como Sahlins (1977), Monaghan (1990 y 1996), Yan (1996), Tereucán (2003 y 2008), Good (2005), Ferraro, (2004), Teigen et al. (2005), Carrasco y Robichaux (2005), Khattri (2010), Peebles (2010), Venkatesan (2011), Mallard (2011), Højer (2012) se han abocado al análisis teórico del tema, poniendo en discusión las premisas de Mauss y/o su estudio en contextos diversos.

Precisamente Yan (1996: 1) en su libro The Flow of Gifts. Reciprocity and Social Networks in a Chinese Village sintetiza una de las ideas centrales contenidas en la generalidad de los estudios, al señalar lo siguiente:

es ampliamente reconocido que el don es uno de los más importantes modos de intercambio social en las sociedades humanas. La obligación de dar y recibir mantiene, fortalece y crea varios vínculos sociales que pueden ser cooperativos, competitivos o antagónicos, permitiendo comprender e interpretar roles culturales y la estructura de relaciones sociales de una determinada sociedad...

Desde una mirada más sociológica, Komter (2005) reafirma también este mismo punto y asigna una función importante al don y/o los intercambios en el mantenimiento de relaciones de solidaridad entre los grupos que componen una sociedad. Complementando a Yan y Komter, debemos agregar la significativa función económica que cumplen los intercambios, especialmente en grupos que tradicionalmente son considerados "pobres" dentro de las mediciones socioeconómicas de los países, como es el caso de los mapuches en Chile.

En tal sentido, la generación de redes o sistemas de intercambios resultan ser relevantes para la subsistencia de los grupos domésticos en un sentido amplio, ya que su análisis debe considerar un conjunto de factores como la organización social, la ritualización de prácticas, las configuraciones espaciales y territoriales, las prerrogativas culturales, entre otros aspectos. 
Por ello, el objetivo del presente trabajo se enfoca, en primer lugar, al análisis de la reciprocidad y el intercambio desde la perspectiva de la intercambiabilidad y procesos asociados en el contexto empírico de análisis, para posteriormente dimensionar los principios involucrados en ella, en especial los referidos a equivalencia y valor puestos en la reciprocidad y el intercambio.

La investigación se realizó en el territorio mapuche o kiñel mapu de Llamuco, conformado por siete comunidades mapuches rurales. Llamuco pertenece a la comuna de Vilcún en la Región de La Araucanía, territorio de localización histórica del pueblo mapuche en el sur de Chile. La extensión territorial es de 1,088.03 hectáreas con una población de 1.094 personas distribuidas en 298 grupos domésticos. Sus habitantes son hablantes de mapudungun (idioma mapuche) y español; sus principales fuentes de ingresos son la pequeña agricultura y la venta de mano de obra en los rubros de la construcción, comercio y servicio doméstico.

El trabajo forma parte de una investigación apoyada por la Universidad de La Frontera, a través de los proyectos DIUFRO núm. DI 09-0065 y DI 09-0066, desarrollados entre 2010 y 2012. El método utilizado fue de tipo etnográfico, para lo cual se desarrolló una labor de campo de diez meses; las principales técnicas de recolección de datos fueron la observación participante y la entrevista en profundidad.

\section{Reciprocidad e intercambio: intercambiabilidad, mercancías y dones}

En palabras de Griesler (2006), las relaciones de intercambio llegan a conformar una estructura de funcionamiento de la sociedad que afecta el conjunto de esferas de vida de las personas, transformándose el don en un hecho social total en términos de Durkheim en la medida que es coercitivo y obligatorio. Plantea además que el don puede integrar al menos de tres elementos teóricos claves: a) distinción social, $b$ ) normas de reciprocidad y c) rituales y simbolismos.

Las distinciones sociales se demostrarían a través de los patrones de interacción entre un dador y un receptor, permitiendo configurar una estructura de relación y condición de las partes involucradas, siendo el parentesco y la amistad dos factores importantes en esta configuración. La norma de la reciprocidad reflejaría el conjunto de reglas y obligaciones que dan paso a un complejo patrón de dar, recibir y devolver, posibilitando además comprender el estándar moral de solidaridad social existente.

El último elemento en el estudio del don son los rituales y simbolismos; estos rituales se definen como actividades gobernadas por reglas de expresión simbólica en donde el don es inculcado a los miembros de un grupo mediante 
una representación colectiva, cuyo fin es el refuerzo de relaciones sociales, en especial entre parientes.

En el contexto empírico de estudio, dichos elementos están estrechamente interrelacionados, ya que las formas de organización social mapuche en Llamuco definen las responsabilidades y compromisos que cada una de las personas tienen con otros, sobre todo parientes patrilineales; las actividades colectivas que involucran el accionar de grupo de parientes; y los tipos de redes territoriales que se construyen mediante los intercambios.

Estas interacciones están lógicamente integradas en normas, prácticas y reglas de equivalencia y valor en los intercambios, que permiten definir los mínimos de conducta de las personas y grupos domésticos en los distintos contextos. Éstos tienen la particularidad de estar incrustados dentro de la cultura, por lo tanto estamos hablando de diferentes tipos de eventos rituales (matrimonios, ritos funerarios, ritos religiosos, etc.), o acciones cotidianas que siguen un estricto protocolo de relación, que le permite dar formalidad y ritualización a los mecanismos de reciprocidad e intercambio.

En tal sentido podemos afirmar que la cultura entre los mapuches actúa como un potente esquema de control social que condiciona y obliga a las familias y grupos a involucrarse en este sistema de redes de intercambio existente en el territorio de Llamuco. No ser parte del mismo es una condición que difícilmente puede darse, ya que a las limitantes económicas para el financiamiento de muchos eventos del ciclo de vida debe agregarse el componente social y de prestigio de las personas y familias. Estos dos aspectos son altamente valorados entre las familias de Llamuco, en especial en rituales con una marcada característica de "público", es decir, que son del escrutinio y evaluación de los participantes y observadores del evento.

Retomando el ciclo de prestaciones involucradas en el don, hay dos elementos que nos permiten situar el análisis en razón de los datos empíricos entre los mapuches. Un primer aspecto refiere a la tercera obligación presente en el ciclo de prestaciones, es decir, el retorno de los dones y el carácter obligatorio de la norma que posibilita la circulación permanente de bienes y servicios dentro de un conjunto de normas simétricas de funcionamiento que regulan los movimientos de transferencia. Un segundo componente es la equivalencia y valor contenido en los mecanismos de reciprocidad e intercambio (Mauss, 1971; Graeber, 2001; Ferraro, 2004; Gudeman, 2001; Gregory, 2009; Peebles, 2010; Godbout, 1997).

Para Mauss, 1971 el intercambio humano comienza con las prestaciones totales; es decir, una afectación al conjunto de la sociedad y en todos sus planos, lo cual lleva a plantear el don como un hecho social total. En esta etapa 
las prestaciones son realizadas entre grupos y adquieren una connotación clasificada dentro del orden de lo no económico, debido a que no es el autobeneficio lo que motiva el intercambio, sino el bienestar del grupo, y por tanto es la norma moral lo que permite la reproducción del ciclo de dar - recibir - devolver.

Toda transacción creará vínculos sociales más allá de las personas, por eso la moralidad emerge de estos vínculos sociales como realidad sui generis de interacciones sociales informales (Mauss, 1971; Godbout, 1997; Mayer, 2002; Hollenbeck et al., 2006; Davies et al., 2010; Mallard, 2011; Kowalski, 2011).

Un segundo estado en el desarrollo de las prestaciones es el intercambio de dones entre individuos que representan a grupos, cambiando el objetivo buscado, ya que los beneficios del intercambio y las relaciones construidas no necesariamente alcanzarían al conjunto de personas que integran el grupo (Godbout, 1997; Tereucán, 2008; Kowalski, 2011).

Finalmente, se llegaría a una tercera etapa marcada por el intercambio de mercancías en las sociedades modernas, y en donde la transacción es realizada entre individuos independientes (Gudeman, 2001; Adloff y Mau, 2006). Para Carrier (1991: 121), Mauss observó dos tipos de intercambios: el del don asociado a aquellas sociedades dominadas por el parentesco; y el de mercancías asociado con la sociedad industrial y dominada por la división de clase y del trabajo.

Sus ideas y postulados han servido de referencia para la generación de una variada discusión de su contenido, de las normas que regulan su funcionamiento, sus clasificaciones y tipologías, sus relaciones con la organización del parentesco, el carácter de los bienes y servicios que entran en circulación, el intercambio dentro de concepciones mercantiles y no mercantiles, los procesos y formas económicas que se dan en determinadas realidades, las formas de capital contenidas dentro de la reciprocidad, entre otros.

Ciertamente la discusión se centra con mayor intensidad en contextos marcados por la presencia de población indígena, ya que en especial la reciprocidad o el don se visualizaba en espacios donde el parentesco constituía el ámbito de relaciones predominantes, y el mercado no tenía una presencia sustancial en la dinámica de estas poblaciones. Aunque en la actualidad es difícil sostener esta premisa para muchas realidades indígenas, la idea de diferenciar entre economías del don marcadas por la moral y la costumbre, y las economías de mercado dirigidas por las transacciones de mercancías, continúan en la discusión y análisis. 
De acuerdo con Lapavitsas (2004: 33) y Gregory (2009), la intercambiabilidad es el concepto tomado para hacer las distinciones entre las características del don y de las mercancías. Éstas representan la racionalidad, el individualismo, el cálculo estricto de beneficio material, las relaciones impersonales y la relación de la propiedad unida al mercado. En contraste, el don representa el poder, la obligación moral, el interés colectivo, las relaciones personales que sobreviven y continúan después del intercambio, un beneficio impreciso y a menudo no material, y una sociedad basada en relaciones abiertas cuyas características son la voluntad y la similitud.

En este campo de análisis, una formulación binaria sobre economía del don versus economía de mercancías es postulada por Gregory, basándose en los conceptos de mercancías de Marx y de dones de Mauss. Para el autor, el intercambio de mercancías es un intercambio de bienes alienables entre contrapartes que están en independencia mutua; mientras que el intercambio de dones es entre cosas inalienables cuyos participantes están en dependencia recíproca.

El intercambio de mercancías establecería relaciones cuantitativas entre los objetos transados, mientras que el intercambio de dones establece relaciones cualitativas entre los sujetos involucrados (Gregory, 1980 y 2009). Al parecer, la intercambiabilidad en las mercancías presentaría una relación más intrínseca, completa y precisa, que la intercambiabilidad de los dones, ya que estos últimos teóricamente no son de manera inherente intercambiables, al estar circunscritos a factores no económicos, como la moral, la religión y la costumbre.

De igual modo, aun cuando los bienes y servicios involucrados en el don se retornen, no existiría precisamente una equivalencia cuantitativa entre las partes al insertarse la prestación en áreas de la vida social que no son inmediatamente tocadas por el mercado: dar para establecer un vínculo y esperar en respuesta un gesto recíproco es fundamental en la relación interpersonal, en las relaciones entre familias, de amistades, laborales, etcétera.

Sin embargo, creemos que el establecimiento de la dicotomía entre don y mercancía o relaciones mercantiles y no mercantiles no necesariamente deben plantearse en el sentido de polos opuestos, ya que en la práctica los intercambios pueden combinar elementos de mercado unido a formas y normas que son más aplicables a relaciones de reciprocidad. Como bien señala Smart (1983: 397), muchos de estos fundamentos necesitan ser cuestionados más que tomados como parte de las definiciones de los dones o mercancías.

Si los dones no son siempre recíprocos y si las motivaciones involucradas en la donación son diversas, lo que es común a los dones como un modo de intercambio puede ser solamente la necesaria forma del don, entendido como las distintas formas de expresarse. 
Lo anterior queda de manifiesto al analizar diversos contextos etnográficos de estudio sobre la temática; por ejemplo, Carrier (1991: 132) señala que los dones y las mercancías no representan categorías exclusivas, sino polos de un continuo, y que muchas transacciones del don contienen un elemento de alienación e individualismo, y muchas transacciones de mercancías tienen obligaciones mutuas.

Por lo tanto, dones y mercancías no son tan fácilmente distinguibles en el caso de mongolian pawnshop estudiado por Højer (2012: 35), fundamento que también es discutido por estudios realizados en Melanesia, como los de: Valeri (1994), Weiner (1985), Parry y Block (1996), Mosko (2000), Graeber (2001) y que nos recuerdan -al menos de forma analítica- la idea de un continuo en los intercambios planteada por Sahlins (1977) en "economía de la edad de piedra” y de los planteamientos que más contemporáneamente establece Lomnitz (2005) sobre reciprocidad negativa, uno de los polos que señalaba Sahlins.

Una visión complementaria es que el intercambio de dones difiere fundamentalmente del intercambio de mercancías, en términos de los roles que caracterizan las relaciones, y en términos de los métodos que las personas usan para incrementar sus ganancias; por lo tanto, cada forma de intercambio debe ser analizada en razón de sus principios esenciales y sus particularidades y cómo la circulación de valores influye en el reino de las mercancías (Bell, 1991a y 1991b; Mayer, 2002; Ferraro, 2004; Teigen et al., 2005; Addo y Besnier, 2008).

Esta postura ofrece una aproximación metodológica interesante para concebir los dones y las mercancías, ya que permitiría comprender los procesos de intercambios en contextos diversos, mediante las prácticas que implementan sus participantes, pero sin abstraerlos de los tipos de interrelaciones existentes, de las formas socioculturales que adoptan muchas de estas transacciones, de las normas y principios que regulan su reproducción, entre otros elementos.

Llevando estas posturas a los datos empíricos entre los mapuches de Llamuco, consideramos que hacer las distinciones entre dones y mercancías es útil para separar analíticamente las diferentes prácticas que familias y comunidades realizan; no obstante, para comprender el funcionamiento de la realidad estudiada, es relevante indagar sobre los contextos sociales y culturales donde los intercambios se dan y se reproducen.

Estos contextos específicos proporcionan los datos para entender las reglas que siguen los participantes, los tipos de bienes que entran en circulación, los flujos de los intercambios, los principios involucrados y los objetivos que estos persiguen. 
De igual modo, se debe considerar en su análisis los elementos de estructura social que fijan el marco de actuación, y con ello nos referimos fundamentalmente a las variables parentesco, residencia y organización social, pues son estos tres elementos los que establecen el juego contextual que "obliga" a la creación de redes y generan los imperativos de la cultura para la producción y reproducción de los mecanismos de reciprocidad e intercambio. En Llamuco, los informantes asocian las mercancías a la existencia del dinero como elemento mediador del intercambio; mientras otras valoraciones y medios utilizados forman parte de la estructura del don.

Con el fin de fijar el análisis específico de la equivalencia y el valor de los intercambios en los apartados siguientes, consideramos necesario delinear una tipología general que permita marcar las diferenciaciones analíticas y los puntos de yuxtaposición existentes.

De este modo, encontramos que entre los mapuches de Llamuco hay intercambios vinculados más directamente con la reciprocidad o el don (rituales vinculados al ciclo de vida o del ciclo de desarrollo de los grupos domésticos, por ejemplo); otros siguen orientaciones más de mercado (transacciones comerciales diversas), y otros presentan características especiales como el trueque, donde visibilizamos una combinación de ambas formas de actuación.

Estas formas no son necesariamente opuestas, sino por el contrario, por lo regular secuenciales y funcionalmente vinculadas unas con otras. $\mathrm{El}$ mayor flujo de intercambios de mercado visualizados en los últimos años en las comunidades de Llamuco, podría hacer suponer una primacía del individualismo y el autointerés en las relaciones que se establecen; pero la observación de las diversas prácticas en las comunidades nos permite constatar la convivencia e interrelación permanente de aquellos intercambios más conceptualizados dentro de la estructura del don o la reciprocidad con los de mercado, lo cual nos lleva a afirmar que un mayor flujo de recursos económicos ha potenciado los tres tipos de intercambios mencionados que se dan entre los grupos domésticos de Llamuco.

\section{Equivalencia y valor en la noción de intercambio y reciprocidad}

Lo equivalente en las formas de intercambio que involucran reciprocidad ha constituido un mecanismo que regula y/o permite la continuidad de una relación, transformándose en el elemento evaluativo del vínculo existente entre personas o grupos. Una regularidad descrita en los estudios sobre el don o la reciprocidad es la tendencia a mantener una relación equilibrada en cuanto a bienes o servicios que se dan y los que regresan. 
En esta dirección, Tereucán (2003: 189), en su estudio sobre reciprocidad entre los nahuas de Tlaxcala, establece que equivalente significa retornar al menos lo mismo que se dio en cantidad y calidad; misma regla que Firth (1974) encuentra entre los maoríes al señalar que todo don debe ser devuelto con un contra-don de un valor como mínimo igual.

Beals (1970: 234-239), en sus estudios con zapotecos de México, señala que los intercambios son altamente formalizados, y la equivalencia en el intercambio es un elemento significativo al momento de la devolución del don dado. En su interpretación, los objetos son considerados como préstamos, y si el retorno no es equivalente, debe compensarse con dinero para igualar su valor cuantitativo.

Una posición ligeramente distinta es la planteada por Purkayastha (2004: 312-313), en su ensayo sobre la teoría de los dones recíprocos, al explicar que existe una obligación en la devolución de determinados intercambios, pero no siempre tienen que ser equivalentes, lo cual genera una ecuación difícil de comprender para los economistas.

Desde la perspectiva del intercambio de mercancías y estrictamente desde la lógica del mercado, el postulado de Purkayastha no es complicado de entender, porque el objetivo en todo intercambio es la obtención de una ganancia; por lo tanto, la asimetría es una condición que siempre se busca.

Distinto es cuando los intercambios son normados por la regla de la equivalencia, pues no recibir al menos lo mismo que se dio pareciera ser un mal negocio, pero aquí entran en juego otros factores relevantes para las partes interactuantes como ser: el contexto específico del intercambio, la relación social existente entre los participantes, las circunstancias económicas puntuales que originaron su no cumplimiento o el esfuerzo realizado por los grupos domésticos por cumplir en parte con el compromiso.

Ahora bien, viéndolo desde un punto de vista práctico, se esperaría que las devoluciones nunca fuesen iguales, ya que al equilibrarse los intercambios la deuda deja de existir. Investigaciones en pueblos del estado de Tlaxcala, México (Tereucán, 2003 y 2008; Carrasco y Robichaux, 2005) señalan que la tendencia en los intercambios que involucran reciprocidad es a devolver lo recibido y agregar otra cantidad, para así mantener el vínculo a través de la deuda. Este mismo hecho es fundamentado también por Godbout (1997: 229-231) al explicar que una deuda de don nunca se liquida, disminuye o se invierte por medio de un don mayor que la deuda, pues la equivalencia es la muerte del don, es una manera de poner fin a una cadena de dones.

De igual modo, es relevante lo indicado por Ferraro (2004: 90) en términos de que la deuda es una categoría cultural que no necesariamente es decidida 
sólo entre dos individuos; y lo establecido por Peebles (2010: 234), en términos de no separar analíticamente en el crédito o la deuda los efectos económicos del beneficio de los componentes morales del mismo.

En muchas circunstancias la valoración del acto de participación puede adquirir un carácter superior a la valorización de la equivalencia en bienes reciprocados, es decir, no se recibe lo mismo que se dio en términos de bienes o servicios, pero sí existe una simetría en términos del acto de reciprocidad. Puesto lo anterior en términos estrictamente económicos no tiene sentido, ya que la inversión realizada no se correspondería con lo recibido; pero visto desde el campo social, sí podemos hablar de una equivalencia, porque el compromiso de relación se cumpliría de la mejor forma posible, y este hecho marca un valor en las relaciones de intercambio, traducido en palabras de los informantes como "respeto", "responsabilidad" y "compromiso de las partes".

Un hecho relacionado con lo anterior y que difícilmente ocurrirá es la negación del don, ya que en todos los casos de intercambios analizados en Llamuco indican que la norma es: una ayuda siempre se recibe, una reciprocidad siempre se acepta, una solicitud de apoyo siempre se evalúa en forma positiva y siempre hay una evaluación de las partes en términos de beneficios mutuos a obtener; por lo tanto, la conducta desinteresada o el don puro en los términos de Mauss no es una categoría presente entre los mapuches, porque todo acto de intercambio involucra una responsabilidad y un compromiso por parte de los involucrados.

Coincidimos en este punto con lo señalado por Cardoso de Oliveira (2004), al postular que el rechazo del intercambio es vivido por el interlocutor como una afirmación de indiferencia o como una agresión (léase, insulto moral), que se expresa con mayor nitidez en el plano de las actitudes o intenciones del agresor, pero también en sus acciones o comportamientos.

Desde otro punto de vista, cuando las equivalencias en los intercambios están más reguladas por las reglas del mercado, lo que se busca siempre es una transacción que satisfaga las necesidades y conlleve un beneficio de ambas partes. Si la condición no se cumple, las partes pueden buscar concretar la transacción con otras personas dentro o fuera de las comunidades, pero no necesariamente implica un quiebre en las relaciones y la continuidad de ayudas o cooperaciones en otros planos, en especial cuando hay factores como el parentesco.

Un ejemplo son las medierías agrícolas en Llamuco, cuyo mecanismo de operación se sintetiza a partir del relato etnográfico de informantes:

Cuando dos personas acuerdan una siembra, uno aportará la tierra y el otro los insumos (semillas, fertilizantes, etc.) compartiendo la mano de obra en el trabajo. Los porcentajes de 
Julio Tereucán-Angulo, Claudio Briceño-Olivera y José Gálvez-Nieto. Equivalencia y valor en procesos de reciprocidad intercambio entre los mapuches

distribución serán comúnmente de $50 \%$ para cada uno, a no ser que medie un acuerdo diferente en virtud de los aportes económicos. Estos acuerdos son establecidos con antelación, ya que es un intercambio bajo las reglas del mercado. No obstante, para llegar a establecer el "trato" debe mediar previamente un conocimiento de las partes y la confianza necesaria que permita asegurar que el "trato" se respetará. Si no se concreta el negocio, se espera que la relación social existente no se altere, prevaleciendo vínculos como el parentesco o la amistad, por lo tanto ambas partes tratarán de disminuir al máximo los niveles de conflictos que pudiesen suscitarse. (Informantes mapuches pertenecientes a la comunidades Juan Antinao Pircunche y José Llancao del territorio de Llamuco).

Otros ejemplos empíricos para denotar las equivalencias en los intercambios de mercado son los conceptos del mapudungun (idioma mapuche), aretun, kakunün y wülatun. El aretun implica la acción de conseguir (pedir prestado) algo a otra persona, pero no existe un pago extra (interés) por parte del beneficiado, sino que devuelve lo mismo. Por ejemplo, si alguien "consigue" un saco de trigo, la equivalencia es la devolución de lo mismo, estipulándose así el plazo de tiempo para la devolución.

Si bien hay un beneficio directo para quien "consigue", queda también de manifiesto una "deuda" que podrá materializarse en similares condiciones bajo la premisa de "vuelta de mano". El kakünun involucra la acción de intercambiar bienes de un mismo tipo (semillas, animales, etc.), con base en un cálculo de equivalencias simétricas, y en donde el precio de mercado es el referente de acción entre las contrapartes.

El wülatun aduce a la venta de un bien o un servicio, es propiamente una relación de mercado en la cual la equivalencia está marcada por la calidad del producto y el precio fijado. El término para comprar, que sería la contraparte de la transacción, es gillan, y la fijación del precio se denomina falin. Esto se condice con lo planteado por Adloff y Mau (2006: 102-103), al señalar que la probabilidad de que las estructuras sociales sobrevivan será mayor, cuando éstas se encuentran conectadas con otras estructuras por relaciones de intercambio recíproco.

En este sentido, la interdependencia sistémica está basada en el patrón de reciprocidad, y la norma puede funcionar como un mecanismo eficiente para comenzar relaciones nuevas o consolidar las ya iniciadas.

\section{Valor en el sistema de intercambios entre los mapuches de Llamuco}

El valor involucrado en los intercambios es un aspecto que se entrelaza en todas las normas y formas en que los intercambios se producen; y al mismo tiempo se 
presenta como un asunto complejo de entender, porque está enraizado en los arreglos sociales y culturales definidos por las partes en el establecimiento de una relación.

Para Narotzky (2004: 98-99) poder determinar cuál es el valor de las cosas, constituye uno de los problemas cruciales que suscita el intercambio. Los bienes que se intercambian deben ser comparables y su tasa de equivalencia aceptada por las partes en el intercambio; sin embargo, la medida de valor no debe confundirse con el valor de uso; el primero es una relación de intercambio y el segundo es un aspecto independiente de éste.

Un elemento que aclara lo que sucede con la cuestión del valor y las lógicas de las personas, es el señalado por Mayer (1974 y 2002) en su estudio con los quechuas de Perú: plantea que cada grupo conoce cuáles son los intercambios generosos, los justos y cuáles son los injustos; y sobre esa base modelarán su comportamiento. Hay circunstancias donde es ventajoso obrar con otras personas de formas más generosas y/o justas, y en otras ocasiones, los intereses propios llevan a obrar de formas injustas.

Desde esta perspectiva, podríamos definir los diferentes grupos que integran un determinado lugar o sociedad y la manera como están relacionadas entre sí estudiando la forma, cantidad, calidad y dirección de los intercambios. Esto constituye un elemento metodológico importante en el análisis de los intercambios, porque permite identificar la dinámica de las relaciones que personas y grupos establecen, posibilitando además configurar intereses individuales, situaciones culturalmente prescritas, obligaciones que van más allá de una reciprocidad, un estricto vínculo comercial, etcétera.

Para determinar este valor, Mayer (1974: 45) afirma que el anfitrión siempre podrá calcular el valor de los bienes que está redistribuyendo o los servicios que está prestando; en tanto, para Bell (1991b: 162) el valor de un don se mide por el valor de la respuesta recíproca. Ciertamente hay intercambios donde es más fácil establecer su valor y, al mismo tiempo, llevar un registro de ellos; pero para las personas de Llamuco esto siempre fue un aspecto difícil de establecer, concluyéndose que una forma de dimensionar el valor de los intercambios es mediante la fórmula: relación de recursos versus utilidad de los mismos en un contexto particular.

$\mathrm{Al}$ parecer, valor de cambio y valor de uso son los dos elementos que parecieran diferenciarse cuando se considera en términos dicotómicos a los dones y mercancías. Godbout (1997: 221-222), refiriéndose al valor en el intercambio, menciona que existe una tendencia a cuantificar la palabra valor y transformarla en una cantidad de dinero; esto es lo que él llama valor de intercambio y que regularmente tenemos por costumbre oponerlo al valor de uso. 
El valor de uso es más cercano a la realidad, pero en la misma medida es único y no representable por medio de una cantidad. Sin desconocer la importancia del valor de cambio y del valor de uso, el autor indica que las cosas adquieren diferentes valores según su capacidad de expresar, de transmitir y de alimentar los lazos sociales, llamando a este vínculo el valor de lazo.

Por lo tanto, este valor no se establece en comparación con otras cosas, sino en relación social entre las personas participantes del intercambio, siendo su objetivo el reforzamiento de los vínculos de cooperación y/o parentesco, y que posibilitará apoyos económicos, sociales o culturales en el futuro. El valor de lazo es el valor simbólico que se relaciona con el don, vinculado con lo que circula en forma de don (Godbout, 1997: 223-224).

A lo anterior, hay que agregar que existen circunstancias en la cuales la retribución de un servicio personal se puede hacer con bienes especificados por "costumbre" y no necesariamente por la devolución del servicio. Ciertamente hay intercambios donde es más fácil establecer su valor y al mismo tiempo llevar un registro exacto de estos bienes, como las prácticas de "ayudas" que describe Monaghan $(1990 ; 1996)$ entre los nuyoocos de Oaxaca, o los intercambios en las fiestas de pueblos en Tlaxcala descritos por Carrasco y Robichaux (2005); pero hay otros que no siempre son fáciles de establecer en el momento de su devolución, como queda de manifiesto en los intercambios de licor en Otavalo, Ecuador, descritos por Barlett (1980), las extensas compensaciones matrimoniales explicadas por Good (2005) o los intercambios en comidas entre mapuches de Argentina y entre los judíos señalados por Kradolfer (2001) y Shuman (2000).

Establecer las relaciones en la práctica pasa por la revisión de los elementos conceptuales que permiten diferenciar diversas prácticas, como señala Bell (1991b): toda relación de intercambio experimenta una equivalencia de valor, definida de alguna manera por los participantes. Es necesario profundizar en estas formas para comprender los tipos de intercambios, los ámbitos que encierra y los factores que influyen en ellos.

Un ejemplo de lo anterior lo conforman los casos en Llamuco cuando la costumbre anula o retrasa la devolución de un intercambio en reciprocidad, sobre todo cuando la ocasión involucra "participar con fuego" en un determinado ritual. Esto se da cuando las mujeres han quedado viudas y con hijos pequeños, o cuando se trata de madres solteras que viven solas con sus hijos menores y su situación económica no les permite cumplir con el compromiso contraído.

1 Asistir a un ritual con licor, refrescos, cervezas y comida para cocinar en el momento, y distribuirlos posteriormente entre los invitados. 
El protocolo demanda que las personas comuniquen esta circunstancia a las contrapartes, lo cual se interpreta como un símbolo de respeto y valoración del vínculo de parentesco o amistad; aunque no significa necesariamente que la deuda desaparezca, pues siempre se esperará alguna retribución. Si a pesar de su situación social y económica deciden participar, el valor del hecho tiene una evaluación especial por quien recibe el beneficio, independientemente de la cantidad de bienes (comida y bebida) que lleven las mujeres al evento. El valor de la acción en este caso es considerablemente mayor y genera un fortalecimiento del vínculo de lazo (Godbout, 1997).

Debemos agregar que estas situaciones no constituyen excepciones a la regla, sino más bien soluciones culturalmente establecidas para remediar, en parte, la rigidez del cumplimiento de compromisos entre familias, en especial aquellas unidas por parentesco patrilineal.

También es necesario establecer y reafirmar que la equivalencia de valor es definida por los participantes, tal y como se señalan en los siguientes ejemplos: a) en caso de defunción de un integrante del grupo doméstico, éste recibirá el apoyo de todos los que forman parte de un kiñe eluwün, ${ }^{2}$ en particular con quienes existe un vínculo de parentesco patrilineal; $b$ ) la valoración de la ayuda es mayor con quienes existe un proceso de cooperación permanente, pero también se espera un apoyo emocional, social o económico más relevante, destacándose el esfuerzo que realizan los grupos domésticos por cumplir con los compromisos adeudados y su sentido de responsabilidad; $c$ ) un ofrecimiento de apoyo voluntario es reconocido como el inicio de vínculos de intercambios, destacándose la acción de la persona o el grupo doméstico más que la cantidad de bienes involucrados en la ayuda.

Los ejemplos denotan las particularidades de la relación entre valor de los bienes involucrados, utilidad de los mismos para quien da y recibe, valor del intercambio y valor de la relación existente; por lo tanto, el análisis de valor lleva consigo todos o casi todos estos elementos, y en virtud de ello es como se dan y establecen las equivalencias en los intercambios.

\section{Intercambios de reciprocidad e intercambios de mercado en Llamuco}

A partir del trabajo de campo es posible asociar algunas características relevantes asociadas a los intercambios referidos a la reciprocidad y otras referidas al intercambio de mercado en Llamuco, lo cual tiene como objetivo más bien una diferenciación de reglas del juego, pues en la práctica muchos

2 Grupo de ayuda integrado por parientes patrilineales y no parientes que tienen como condición la cercanía de residencia en una comunidad. 
de los intercambios de mercado no pueden analizarse sin considerar el contexto de relaciones donde los participantes están involucrados. En Llamuco, las equivalencias en los intercambios que involucran reciprocidad no siempre guardan una simetría, aun cuando los participantes conocen bien cuánto de un determinado bien o servicio es apropiado dar y cuánto se espera recibir en un compromiso.

Pero para entender esta forma de actuar es necesario considerar el contexto sociocultural donde estos intercambios se producen, lo cual se refleja en el siguiente caso: todo grupo doméstico forma parte de un xokinche, o grupo de parentesco patrilinealmente localizado. La pertenencia al mismo lleva consigo la responsabilidad de apoyar social y económicamente a los parientes patrilineales, sobre todo hermanos, padres, tíos e hijos.

Para el caso específico de una boda, un informante hombre de 60 años explica el proceder en términos de sus compromisos:

Yo tengo tres hijos hombres, pero uno de mis hermanos tiene dos hijos hombres y dos mujeres y mi otro hermano tiene tres hijas. Cuando las hijas de mis hermanos se casen, yo tengo la responsabilidad de contribuir económicamente a la realización del mafutün (matrimonio mapuche) de todas ellas, por lo que deberé hacer un gasto importante. No obstante, como yo solamente tengo hijos hombres el nivel de reciprocidad conmigo no será el mismo, debido a que los padres deben gastar más dinero cuando se casan las hijas, pero eso es algo que yo sé que es así.

En este caso no hay una equivalencia simétrica en la devolución del gasto, pero se asume como correcto porque es parte de la "responsabilidad" por pertenecer al mismo xokinche. No obstante, indica: "Probablemente mis hermanos sean más 'generosos' conmigo cuando yo requiera su apoyo en otro tipo de situaciones...”.

Este mismo hecho es replicable en el caso de defunciones. En Llamuco todos los parientes patrilineales forman parte de un kiñe eluwün, lo cual conceptualmente implica que se actúa como grupo en un ritual de muerte. En tal caso, cada integrante del grupo patrilineal apoyará social, emocional y económicamente a quienes han sufrido la pérdida de un familiar durante todo el rito funerario. Por lógica, aquí no existe una cuantificación del número de veces que a alguien le ha correspondido apoyar, pues lo relevante es el soporte del grupo. Una regla que señalan todos los informantes es que cuando se da algo siempre se espera recibir al menos lo mismo en cantidad y calidad.

De hecho, los informantes explican que llevan registros escritos de quiénes los han apoyado, con qué bienes o servicios, en qué cantidades y cuántas veces. La equivalencia equilibrada o simétrica pareciera ser la norma operante; pues la asimetría puede implicar un deterioro en la relación o su rompimiento, 
aunque en los casos observados las personas siempre indicaron que había que mantener la deuda, por lo tanto: o se daba más en cantidad o se mejoraba la calidad de los bienes en circulación en los intercambios.

En este sentido, observamos al menos seis consideraciones que reflejan los contextos donde los intercambios fluyen: a) cuando un grupo doméstico joven está participando por primera vez en algún ritual (matrimonios, ritos funerarios o ritos religiosos) marca un referente de equivalencia que espera sea devuelto en las mismas condiciones en el futuro; $b$ ) cuando se está devolviendo una ayuda, el organizador del ritual espera una equivalencia como medida de reciprocidad, y evalúa la participación y su relación con el grupo doméstico en virtud de los bienes económicos que entran en circulación; $c$ ) cuando un grupo doméstico - por diversas circunstancias- no puede responder de la misma forma a otro (cantidad y calidad de lo recibido), deberá dar las explicaciones correspondientes. En tal caso, se valorará el esfuerzo que las personas realizan por cumplir el compromiso y no se produce una fractura en la relación social existente; $d$ ) cuando las personas se ven imposibilitadas para retribuir la ayuda, siempre debe existir una causa que culturalmente lo justifique, como es la situación de mujeres que han enviudado recientemente y tienen aún hijos pequeños; $e$ ) cuando los intercambios involucran servicios (horas o días de trabajo) solicitados, la equivalencia siempre se presenta como una condición obligatoria por cumplir, ya sea por quien recibió el beneficio en primera instancia u otra persona que éste consiga para actuar en su lugar, pero siempre debe existir una respuesta simétrica; y $f$ ) cuando se trata de intercambios que involucran más las reglas del mercado, las equivalencias no siempre son simétricas, pues el beneficio individual es lo que está detrás de la transacción. No obstante, dicha relación siempre se encuentra mediada por un acuerdo inicial que las partes han aceptado.

Visto lo anterior desde las reglas de la equivalencia, tenemos que en ambas transacciones debe existir un equilibrio de valor definido de alguna forma por los participantes, y se podría pensar que un intercambio justo o equilibrado implica un aumento de la utilidad en forma igualitaria para cada una de las partes, satisfaciendo la regla de la equivalencia para uso general.

Sin embargo, la equivalencia de valor puede tener variadas interpretaciones y las formas en que se soluciona una disconformidad pueden presentar también modalidades diferentes; pero si las mercancías se ven solamente como fenómeno económico, no se logrará comprender la totalidad de la perspectiva de la intercambiabilidad, ya que las mercancías son también productos útiles, y su utilidad interactúa con el valor de intercambio en el curso de las transacciones que se realizan. Estos dos componentes - equivalencia y valor-son 
relevantes para la comprensión del intercambio, la reciprocidad y sus relaciones con el mercado, principalmente porque fijan pautas de relación y formas de actuación de los participantes.

\section{Conclusiones}

Tomando los postulados de los autores y examinándolos con los datos de campo en comunidades mapuches de Llamuco, señalamos la necesidad de hacer las separaciones tácitas entre formas de intercambio que involucran las reglas del mercado, y otras en donde prima la reciprocidad o el don, pero sin ser demasiado categóricos en el establecimiento de la dicotomía, pues en muchas ocasiones ambas formas están fusionadas, al menos en las reglas que actúan para la generación de la relación.

En el contexto empírico de investigación, las personas distinguen claramente los intercambios que conllevan reciprocidad de otras formas de relaciones. Términos como "compromiso" y "responsabilidad" denotan una relación basada en la ayuda mutua, donde el vínculo de parentesco y amistad son componentes esenciales en el intercambio generado, y la equivalencia como norma de operación es lo que está implícito en los acuerdos establecidos. Por otro lado, para los intercambios que involucran "dinero", los conceptos que se usan son "negocio" y "deuda" y, aunque las reglas del mercado en términos de beneficios económicos es lo que prima, también encontramos un matiz importante de normas que regulan las relaciones de intercambio recíproco en este tipo de transacciones.

Lo anterior está directamente relacionado con la condición de los participantes, debido a que si la relación se da entre personas de una misma comunidad o dentro del territorio de Llamuco, éstas procurarán no sacar un beneficio individual a costa de perjudicar al otro, sino más bien establecer una relación "justa" para ambas partes.

La precaución no se basa exclusivamente en estrictas normas morales, sino más bien está condicionada a los procesos de interacción permanente que las personas y los grupos domésticos tienen en Llamuco, y a la necesidad de no romper con redes de relaciones que pueden ser útiles en diferentes momentos; por lo tanto, evitar un conflicto por transacciones que no conllevan un gran beneficio individual parece ser una conducta apropiada.

Financiar determinados hechos, por ejemplo bodas, ritos funerarios o rituales religiosos, involucra un costo económico que muchas veces un grupo doméstico no está en condiciones de asumir por cuenta propia, y para lograrlo necesita la presencia de otros para contribuir en su ejecución. Sin embargo, 
estos apoyos no se consiguen por caridad o solidaridad desinteresada, sino se requiere ser parte de redes que proporcionen este nivel de seguridad, e implica niveles de reciprocidad y equivalencia más o menos similares, para evitar así un conflicto con algunos de los participantes.

En este sentido, se debe considerar que los intercambios en Llamuco no funcionan como mecanismos aislados de relación entre personas, sino que integran un conjunto de relaciones fundadas en el parentesco, en la residencia y en la organización social; por lo tanto, muchos de los intercambios mercantiles están basados en relaciones de reciprocidad anteriores y la confianza necesaria para establecer acuerdos de beneficio económico mutuo.

También existen otras modalidades de intercambios vinculados al establecimiento de formas diádicas que se pueden situar dentro de formas comerciales de relación como el aretun (conseguir), kakünun (cambiar), wülatun (vender) y xafkintu (trueque). La particularidad de ellos es que se pueden relacionar analíticamente a modalidades de intercambios de mercado, pero los límites de separación tácita entre prestaciones y mercado no siempre son tan visibles.

Más que diferenciar si el intercambio es de mercado o se rige por las normas de la reciprocidad, es importante identificar y comprender el contexto donde se dan los intercambios. En el análisis de la reciprocidad encontramos características de mercado como la asignación de valor y calidad de acuerdo con precios de determinados productos, y en los intercambios mercantiles hallamos que muchos "negocios" o acuerdos productivos se dan en un marco de confianza, generado por las relaciones de reciprocidad.

Por tanto, para entender el intercambio y la reciprocidad no sólo debe tomarse en cuenta la construcción de relaciones sociales, sino también la de relaciones económicas, dado que lo económico es relevante en Llamuco para sostener y reproducir este tipo de prácticas en las comunidades y apoyar los propias estrategias económicas de los grupos domésticos.

\section{Referencias}

Addo, Ping-Ann y Niko Besnier (2008), "When gifts become commidities: Pawnshops valuables, and shame in Tonga and the Tongan diáspora", en Journal of the Royal Anthropological Institute, vol. 14, Reino Unido: Royal Anthropological Institute.

Adloff, Frank y Stephen Mau (2006), "Giving social ties, reciprocity in modern society", en European Journal of Sociology, vol. 47, núm.1, Cambridge: Cambridge University Press.

Barlett, Peggy (1980), "Reciprocity and the San Juan Fiesta”, en Journal of Anthropological Research, vol. 36, núm. 1, Estados Unidos: University of New Mexico. 
Julio Tereucán-Angulo, Claudio Briceño-Olivera y José Gálvez-Nieto. Equivalencia y valor en procesos de reciprocidad intercambio entre los mapuches

Beals, Ralph (1970), "Gifting, reciprocity, saving and crédito in Peasanta Oaxaca”, en Southwestern Journal of Anthropology, vol. 26, núm. 3, Estados Unidos: University of New Mexico.

Bell, Duran (1991a), "Reciprocity as a generating process in social relations", en Journal of Quantitative Anthropology, núm. 3, Netherland: Kluwer academic publishers.

Bell, Duran (1991b), "Modes of Exchange: Gift and Commodity", en The Journal of SocioEconomics, vol. 20, núm. 2, Netherland: JAI Press Inc.

Bourdieu, Pierre (2007), El sentido práctico, Argentina: Siglo XXI.

Cardoso de Oliveira, Luis (2004), "Honor, dignidad y reciprocidad", en Cuadernos de Antropología Social, núm. 20, Argentina: Universidad de Buenos Aires.

Carrasco, Guillermo y David Robichaux (2005), "Parentesco, compadrazgo y ayuda: el caso de las fiestas quiceañeras en Tlaxcala”, en Robichaux, David [ed.], Familia y parentesco, en México y Mesoamérica. Unas miradas antropológicas, México: Universidad Iberoamericana.

Carrier, James (1991), "Gifts, Commodities and Social Relations: A Maussian View of Exchange”, en Sociological Forum, vol. 6, núm. 1, Estados Unidos: Eastern Sociological Society.

Cheal, David (1988), The gift economy, Nueva York, Londres: Routledge.

Davies, Gary et al. (2010), "Gifts and Gifting", en International Journal of Management Review, vol. 12, Reino Unido: British Academy of Management and Blackwell Publishing Ltd.

Ferraro, Emilia (2004), "Owing and being in debt. A contribution from the northern Andes of Ecuador”, en Social Anthropology, vol. 12, núm. 1, Reino Unido: European Association of Social Anthropologists.

Firth, Raymond (1974), Temas de Antropología Económica, México: Fondo de Cultura Económica.

Godbout, Jacques (1997), El espiritu del don, México: Siglo XXI.

Good, Catharine (2005), "Trabajando juntos como uno: conceptos nahuas del grupo doméstico y la persona”, en Robichaux, David [ed.], Familia y parentesco en México y Mesoamérica. Unas miradas antropológicas, México: Universidad Iberoamericana.

Graeber, David (2001), "Marcel Mauss Revisited”, en Graeber, David [ed.], Toward and Anthropological Theory Value, Estados Unidos: Palgrave Macmillan.

Gregory, Christhopher (1980), "Gifts to men and gifts to god: Gift exchange and capital accumulation in contemporary Papu New Guinea”, en Man, núm. 15, Reino Unido: Royal Anthropological Institute of Great Britain and Ireland.

Gregory, Christhopher (2009), “Whatever happened to economic anthropology?”, en The Australian Journal of Anthropology, vol. 20, Australia: Australian Anthropological Society.

Griesler, Markus (2006), “Consumer gift system: Netnographic insights from Napster”, en Journal of consumer research, Estados Unidos: The University of Chicago Press Journals.

Gudeman, Stephen (2001), Economy's tension: the dialectics of community and market, Estados Unidos: Berghahn Books.

Højer, Lars (2012), “The spirit of bussines: Pawnshop in Ulaanbaatar”, en Social Anthropology/Antropologie sociale, vol. 20, Reino Unido: European Association of Social Anthropologists. 
Hollenbeck, Candice et al. (2006), "Gift giving: A community Paradigm”, en Psichology \& Marketing, vol. 23, Estados Unidos: Wiley Periodicals.

Khattri, Man (2010), "Rites of Passage: Flow of Gifts", en Dhaulagiri Journal of Sociology and Anthropology, vol. 4, Nepal: Departament of Sociology/ Antrhropoly, Tribhuvan University.

Kradolfer, Sabine (2001), "La importancia del don como fundamento para las relaciones sociales en las comunidades mapuche de Argentina”, en Scripta Ethnológica, vol. XXIII, Argentina: Centro Argentino de Etnología Americana.

Komter, Aafke (2005), Social Solidarity and the Gift, Nueva York: Cambridge University Press.

Kowalski, Robert (2011), “The Gift - Marcel Mauss and international aid”, en Journal of Comparative Social Welfare, vol. 27, núm. 3, Reino Unido: Routledge, Taylor andd Francis Group.

Lapavitsas, Costas (2004), "Commodities and Gifts: Why Commodities Represent More than Market Relations”, en Science \& Society, tomo 68, núm. 1, Estados Unidos: Guilford Publications.

Lomnitz, Claudio (2005), "Sobre la reciprocidad negativa", en Revista de Antropología Social, vol. 14, España: Universidad Complutense de Madrid.

Mallard, Grégoire (2011), “The Gifts revisited: Marcel Mauss on War, Debt, and the Politics of Reparations”, en Sociological Theory, vol. 29, Estados Unidos: American Sociological Association.

Mauss, Marcel (1971), Sociología y Antropología, España: Tecnos.

Mayer, Enrique (1974), "Las reglas del juego en la reciprocidad andina”, en Alberti Georgio et al. [comps.], Reciprocidad e intercambio en los andes peruanos, Perú: Instituto de Estudios Peruanos.

Mayer, Enrique (2002), Articulated peasant. Household economies in the Andes, Estados Unidos: Westview Press.

Monaghan, John (1990), "Reciprocity, Redistribution, and the Transaction of Value in the Mesoamerican Fiesta”, en American Ethnology, vol. 17, núm. 4, Estados Unidos: American Anthropological Association.

Monaghan, John (1996), "Fiesta finance in Mesoamerica and the origins of a gift exchange system”, en The Journal of the Royal Anthropological Institute, vol. 2, núm. 3, Reino Unido: Royal Anthropological Institute of Great Britain and Ireland.

Mosko, Mark (2000), "Inalienable Ethnography: Keeping-While-Giving and the Trobriand Case", en The Journal of the Royal Anthropological Institute, vol. 6, núm. 3, Reino Unido: Whiley-Blackwell on behalf of the Royal Anthropological Institute of Great Britain And Ireland.

Narotzky, Susana (2004), Antropología económica. Nuevas tendencias, España: Melusina.

Parry, Jonathan y Maurice Block (1996), Money \& the morality of exchange, Reino Unido: Cambridge University Press.

Peebles, Gustav (2010), "Anthropology and credit and debt", en Annual review od Anthropology, vol. 39, Estados Unidos: Annual Reviews.

Purkayastha, Dipankar (2004), "A theory of reciprocal gifts", en Atlantic Economical Journal, vol. 32, núm. 4, Estados Unidos: Atlantic Economical Society.

Sahlins, Marshall (1977), Economia de la edad de piedra, Madrid: Akal. 
Shuman, Amy (2000), "Food gifts: ritual exchange and the production of excess meaning", en The Journal of American Folklore, vol. 113, núm. 450, Estados Unidos: University of Illinois Press.

Smart, Alan (1983), "Gifts, Bribes and Guanxi: a reconsideration of Bourdieu's social capital”, en Cultural Anthropology, vol. 8, núm. 3, Estados Unidos: Society for Cultural Anthropology.

Teigen, Karl et al. (2005), “Giver-receiver asymmetries in gift preferences”, en British Journal of Social Psychology, vol. 44, núm. 1, Reino Unido: The British Psychological Society.

Tereucán, Julio (2003), "Redes de reciprocidad e intercambio y su importancia en la cultura y economía de una comunidad postnahua de Tlaxcala”, Tesis de Maestría en Antropología Social, México: Universidad Iberoamericana.

Tereucán, Julio (2008), "Reciprocidad e intercambio entre los mapuches: organización social y economía en comunidades rurales del sur de Chile”, Tesis doctoral en Antropología Social, México: Universidad Iberoamericana.

Valeri, Valerio (1994), "Buying women and selling them: gift and commodity exchange in Huaulu alliance”, en Man, vol. 29, núm. 1, Reino Unido: Royal Anthropological Institute of Great Britain and Ireland.

Venkatesan, Soumhya (2011), "The social life a 'free' gift", en Journal of the American Ethnological Society, vol. 38, Estados Unidos: American Anthropological Association.

Weinner, Annette (1985), "Inalienable Wealth", en American Ethnologist, vol. 12, núm. 2, Estados Unidos: American Ethnological Society.

Yan, Yunxiang (1996), The Flow of Gifts. Reciprocity and Social Networks in a Chinese Village, Estados Unidos: Standford University Press.

Julio César Tereucán Angulo. Doctor en Antropología Social por la Universidad Iberoamericana de México. Académico e Investigador del departamento de Trabajo Social y del Centro de Investigaciones Territoriales de la Universidad de La Frontera, Temuco, Chile. Líneas de investigación: economía cultural, organización social indígena, políticas públicas. Publicaciones recientes: Tereucán Julio y Claudio Briceño, "Estado, política social y desarrollo territorial local. Análisis del concepto de región de refugio", en Revista Chilena de Estudios Regionales, núm. 1 (2009); Gálvez José, Julio Tereucán, Sergio Muñoz, Claudio Briceño y Mayorga Muñoz, "Propiedades psicométricas del cuestionario para evaluar clima social del centro escolar (CECSCE)", en LIBERABIT Revista de Psicología (Scielo), vol. 20, núm. 1 (2014); Robles Carmen y Julio Tereucán, "Efectividad de la medida de libertad vigilada. Experiencia del Centro de Reinserción Social de Angol”, en Revista Cuadernos de Trabajo Social, vol. 7, núm. 1, Latindex (2015). 
Claudio Andrés Briceño Olivera. Magister en Investigación y Desarrollo Local por la Universidad Complutense de Madrid. Académico e Investigador del departamento de Trabajo Social. Líneas de investigación: políticas públicas y sociales, pobreza, economía informal. Publicaciones recientes: Briceño Claudio, Hilda Llanquinao y Olga Rebolledo, "Traditional Mapuche education. Contribution for an Intercultural Education Journal of Intercultural Communication”, issue 16 (2006); Briceño Claudio, Hilda Llanquinao y Olga Rebolledo, "El Pentukum como metodología de enseñanza-aprendizaje en la cultura mapuche", en Revista de Lengua y Literatura Mapuche (2008); Tereucán Julio y Claudio Briceño, "Estado, política social y desarrollo territorial local. Análisis del concepto de región de refugio", en Revista Chilena de Estudios Regionales, núm. 1 (2009).

José Luis Gálvez Nieto. Magister en Gerencia Social por la Universidad de La Frontera de Chile. Académico e Investigador del departamento de Trabajo Social. Líneas de investigación: educación, políticas públicas y sociales. Publicaciones recientes: Gálvez-Nieto J. L., D. Vera-Bachmann e I. Trizano, "Estudio confirmatorio del Cuestionario para evaluar Clima Social del Centro Escolar en Chile”, en Revista Mexicana de Psicología, vol. 32, núm. 2 (2015); Gálvez-Nieto J. L., D. Vera-Bachmann, I. Trizano y J. A. García, "Examen psicométrico de la Escala de Actitudes hacia la Autoridad Institucional (AAI-A), en estudiantes chilenos", en Revista Iberoamericana de Diagnóstico y Evaluación Psicológica, vol. 39, núm. 1 (2015); Gálvez-Nieto J. L., D. Vera-Bachmann, C. Cerda y R. Fuentes, "Escala de victimización entre adolescentes a través del teléfono móvil y de internet: estudio de validación de una versión abreviada de 10 ítems", en Revista Iberoamericana de Diagnóstico y Evaluación Psicológica, vol. 41, núm. 1 (2016); Gálvez-Nieto J. L., K. Polanco y S. Salvo, "Propiedades psicométricas de la Escala de Autoconcepto Académico (EAA) en estudiantes chilenos", en Revista Iberoamericana de Diagnóstico y Evaluación Psicológica (en prensa).

Recepción: 30 de septiembre de 2015.

Aprobación: 18 de julio de 2016. 